\title{
Research on the Small Deformation of Thin Arched Plates in Fluids
}

\author{
Junxi Bi a, Bin Liu ${ }^{b}$ \\ Inner Mongolia University of Technology, Hohhot 010051, China \\ a2755178674@qq.com, b3144685951@qq.com
}

\begin{abstract}
Keywords: Fluid-Solid Coupling, Offshore Pipeline Plough's Fender, United Lagrange-Euler Method, A Thin Arched Plate, Bending Deformation.
\end{abstract}

\begin{abstract}
This paper applied the theory of thin elastic shell and fundamental equation of fluid mechanics, combined with united Lagrange-Euler method, to establish the kinematic equation and dynamic equation of contact surfaces between fluid and solid. The linear equations of plate's normal displacement coefficients under incline and symmetrical flow were given. Based on the boundary conditions, the expressions of plate's deformation, pressure and stress were also derived. By examples, some deformation curves under different materials, thicknesses, arc lengths, angles of attack and fluid velocities were plotted; the positions where maximum deformation and maximum stress occurred as well as the size was discussed. In addition, the influence of different parameters on plate's deformation and stress was analyzed.
\end{abstract}

\section{Introduction}

Offshore pipeline ploughs are used for laying pipelines in seabed trenches, which primarily consist of a plough body, fenders, skid and steering systems [1]. The function of fenders is to realize the return of mud into trenches. For the best results, it is essential to study its stress and deformation under the influence of fluid. In the theoretical analysis, a fender can be described as a thin arched plate that is able to be studied by using hydroelasticity theory [2]. The theory of hydroelasticity is an important branch of learning for studying the interaction between liquid, gas and elastic structure and has increasingly become the main research object in many engineering fields. With the development of computing methods and an imperious demand in engineering fields, the solution for fluid-structure interaction has made considerable progress in both theoretical research and engineering applications, especially in ocean engineering [3].

In the many study fruits, the study on elastic solid deformation and internal forces analysis in fluidstructure coupling is very little. However, a thin arched plate has already attracted broad attention due to its special structure. On the basis of theory of shells and basic equation of fluid dynamics given in [4], by united Lagrangian-Eulerian method is provided a detailed analysis on bending deformation and stress of the thin elastic plate in a continuous incompressible ideal cross-flow. The results provide a theoretical basis for the strength and stiffness design of thin elastic plates in fluid [5].

\section{Establishment of fundamental equations}

In $r \theta z$ cylindrical coordinate system established in Fig. 1a, where $\mathrm{o}$ is ordinate origin and $\mathrm{z}$ is along the axial line of a thin arched plate, an unlimited length plate is a part of shell's edges and hit by a continuous incompressible ideal cross-flow, shown in Fig. 1b. The pressure, intensity and velocity of the fluid are defined as $p_{\infty}, \rho_{\infty}$ and $v_{\infty} . \theta_{0}$ is the angle between symmetry axis of the cross section and direction of flow. Assuming that the internal pressure of the plate $p^{*}$ remains constant. In steady flows, the velocity potential function of fluids $\varphi$ is determined by the flowing equations[6]:

$$
\left\{\begin{array}{c}
\nabla^{2} \varphi=0 \\
p=p_{\infty}+\frac{\rho_{\infty}}{2}\left[v_{\infty}^{2}-(\nabla \varphi)^{2}\right] \\
\varphi=-v_{\infty} r \cos \theta \quad(r \rightarrow \infty)
\end{array}\right.
$$


where $\nabla=\frac{\partial}{r \partial \theta}+\frac{\partial}{\partial r}, \nabla^{2}=\frac{\partial^{2}}{r^{2} \partial \theta^{2}}+2 \frac{\partial^{2}}{r \partial \theta \partial r}+\frac{\partial^{2}}{\partial r^{2}}, p$ is fluid pressure.

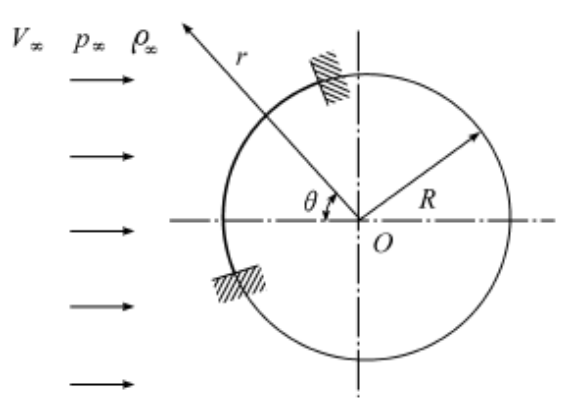

1a)

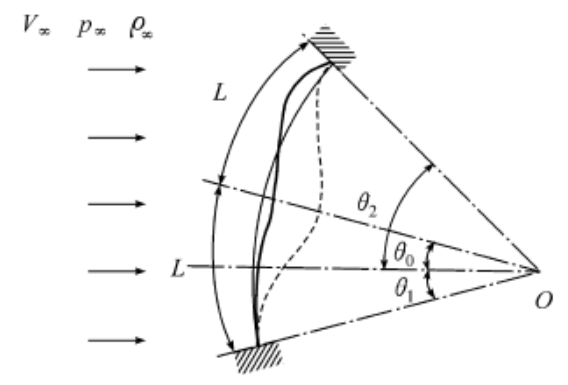

1b)

Fig. 1 Transversal cross-flow on arc shell

The kinematic and dynamic constraints on the plate can be regarded as:

$\frac{\partial \varphi}{\partial r}=\frac{1}{r^{2}} \frac{\partial w}{\partial \theta} \frac{\partial \varphi}{\partial \theta}-w \frac{\partial^{2}}{\partial r^{2}}+\frac{v}{r} \frac{\partial^{2} \varphi}{\partial r \partial \theta} \quad(r=R)$

$Z_{\theta}=0 \quad Z_{r}=p^{*}-p-w \frac{\partial p}{\partial r}-\frac{v}{r} \frac{\partial p}{\partial \theta} \quad(r=\quad R)$

where $\mathrm{w}$ and $\mathrm{v}$ represent the normal displacement and the tangential displacement; $\mathrm{Z}_{\mathrm{r}}$ and $\mathrm{Z}_{\theta}$ are the components of external force in the direction of $r$-axis and $\theta$-axis. After simplifying the small deformation in a thin arched plate, we obtain linear equations for internal force[6]:

$$
\left\{\begin{array}{c}
\frac{\partial N_{\theta \theta}}{\partial \theta}+\frac{Q_{\theta \theta}}{R}=0 \\
\frac{\partial Q_{\theta \theta}}{\partial \theta}-N_{\theta \theta}+R Z_{r}=0 \\
\frac{\partial M_{\theta \theta}}{\partial \theta}-R Q_{\theta \theta}=0 \\
M_{\theta \theta}=-\frac{D}{R^{2}} \frac{\partial^{2} w}{\partial \theta^{2}}
\end{array}\right.
$$

where $\mathrm{N}_{\theta \theta}$ and $\mathrm{Q}_{\theta \theta}$ are mid-surface stress, $\mathrm{M}_{\theta \theta}$ is the moment of internal forces, $\mathrm{D}$ is bending rigidity and $\mathrm{R}$ is the radius of mid-surface.

Putting the last formula in equations(4) into three other formulas to eliminate $M_{\theta \theta}, Q_{\theta \theta}, N_{\theta \theta}$, then combined with formula(3), we get:

$$
\frac{\partial^{4} w}{\partial \theta^{4}}+\frac{\partial^{2} w}{\partial \theta^{2}}=\frac{R^{4}}{D}\left[p^{*}-p-w \frac{\partial p}{\partial r}-\frac{v}{r} \frac{\partial p}{\partial \theta}\right] \quad(r=R)
$$

By rigid fixation on both ends, the boundary condition is expressed as:

$v=w=\frac{\partial w}{\partial \theta}=0 \quad\left(\theta=\theta_{1}, \theta_{2}\right)$ $1 b$.

where $\theta_{1}$ and $\theta_{2}$ are angles between water-flow and radiuses of the arc at the ends, shown in Fig.

\section{Solution of deformation and stress}

\subsection{The general solution of a thin arched plate under the influence of fluids}

Fluid potential $\varphi_{1}$ and pressure $p_{1}$ were introduced to study the interaction between fluids and rigid plates. As for thin elastic plates, there exist disturbance potential $\varphi_{2}$ and pressure $p_{2}$ caused by displacement of the interface, so we have:

$$
\varphi=\varphi_{1}+\varphi_{2} \quad p=p_{1}+p_{2} \quad w=w_{-} 2
$$


substituting the formula (7) into formulas (1), (2), (4), (5), then we get[7]:

$$
\begin{aligned}
& \varphi_{1}=-v_{\infty}\left(r+\frac{R^{2}}{r}\right) \cos \theta \\
& p_{1}=p_{\infty}-\frac{\rho_{\infty} v_{\infty}^{2}}{2}\left(\frac{R}{r}\right)^{2}\left[\frac{R^{2}}{r^{2}}-2 \cos (2 \theta)\right]
\end{aligned}
$$

The expressions $\varphi_{2}, \mathrm{p}_{2}$, w satisfy the following equations:

$$
\begin{aligned}
& \nabla^{2} \varphi_{2}=0 \\
& \varphi_{2}=0 \quad(r \rightarrow \infty) \\
& \frac{\partial \varphi_{2}}{\partial r}=\frac{1}{r^{2}} \frac{\partial w}{\partial \theta} \frac{\partial \varphi_{1}}{\partial \theta}-w \frac{\partial^{2} \varphi_{1}}{\partial r^{2}} \quad\left(r=R, \theta_{1}<\theta<\theta_{2}\right) \\
& \frac{\partial \varphi_{2}}{\partial r}=0 \quad\left(r=R, \theta_{2} \leq \theta \leq 2 \pi+\theta_{1}\right) \\
& p_{2}=-\rho_{\infty}\left(\frac{\partial \varphi_{1}}{\partial r} \frac{\partial \varphi_{2}}{\partial r}+\frac{1}{r^{2}} \frac{\partial \varphi_{1}}{\partial \theta} \frac{\partial \varphi_{2}}{\partial \theta}\right) \\
& \frac{\partial^{4} w}{\partial \theta^{4}}+\frac{\partial^{4} w}{\partial \theta}=\frac{R^{4}}{D}\left(p^{*}-p_{1}-p_{2}-w \frac{\partial p_{1}}{\partial r}-\frac{v}{r} \frac{\partial p_{1}}{\partial \theta}\right) \quad\left(r=R, \theta_{1}<\theta<\theta_{2}\right)
\end{aligned}
$$

In the process of solving $\varphi_{2}, \mathrm{p}_{2}, \mathrm{w}$, the function $\mathrm{w}$ which meets the boundary condition (6) can be expressed as[7]:

$$
\begin{aligned}
& w=-\frac{L^{2}}{R}\left\{\sum_{i=1}^{I} f_{i}\left[1+(-1)^{i-1} \cos (i \pi \xi)\right]+\sum_{j=1}^{J} q_{j} \sin (j \pi \xi) \cos \left(j \frac{\pi}{2} \xi\right)\right\} \\
& (i=1,2, \ldots, I ; j=1,2, \ldots, J)
\end{aligned}
$$

where $\xi=\left(\theta-\theta_{0}\right) \frac{R}{L} ; \mathrm{f}_{\mathrm{i}}, \mathrm{q}_{\mathrm{j}}$ are constants, $\mathrm{L}$ is the length of semi - arc.

If a thin arched plate is laid at an arbitrary angle, the velocity potential function meeting the formulas $(10) \sim(13)$ can be written as[8]:

$$
\varphi_{2}=\sum_{n=1}^{N}\left\{\left(\frac{R}{r}\right)^{n}\left[A_{n} \cos (n \theta)+B_{n} \sin (n \theta)\right]\right\} \quad(n=1,2, \ldots, N)
$$

We can put the expressions (8) and (17) into the formulas (12) and (13) and integrated the result by Bubnov - Galerkin Method, then we get:

$$
\int_{0}^{2 \pi} \frac{\partial \varphi_{2}}{\partial r}\left[\begin{array}{c}
\cos (n \theta) \\
\sin (n \theta)
\end{array}\right] d \theta=\int_{\theta_{1}}^{\theta_{2}}\left[\frac{\partial w}{r^{2} \partial \theta} \frac{\partial \varphi_{1}}{\partial \theta}-w \frac{\partial^{2} \varphi_{1}}{\partial r^{2}}\right] \times\left[\begin{array}{c}
\cos (n \theta) \\
\sin (n \theta)
\end{array}\right] d \theta
$$

According to (8), (17) and (14), when $\mathrm{r}=\mathrm{R}, \mathrm{p}_{2}$ is:

$$
\begin{aligned}
& \quad p_{2}=\frac{2 \rho_{\infty} v_{\infty}^{2} l^{2}}{\pi} \sum_{n=1}^{N}\left\{\left[\sum_{i=1}^{l} C_{n i} f_{i}+\sum_{j=1}^{J} D_{n j} q_{j}\right] \times[\cos (n-1) \theta-\cos (n+1) \theta]+\right. \\
& \left.\left(\sum_{i=1}^{I} F_{n i} f_{i}+\sum_{j=1}^{J} G_{n j} q_{j}\right)[\sin (n-1) \theta-\sin (n+1) \theta]\right\} \quad(n= \\
& 1,2, \ldots, N)
\end{aligned}
$$

In case of neglecting the effect of hydrostatic pressure coefficients, we introduce the fluid elastic mechanical parameter $\mu$.

$$
\mu=\frac{\rho_{\infty} \mathrm{v}_{\infty}^{2} \mathrm{RL}^{2}}{2 \mathrm{D}}
$$

By substituting expression (9), (16), (18) into expression (15), it can obtain the expressions of coefficients $f_{i}, q_{i}$ of normal deflection $w$ using Galerkin integral method, with first approximation being set $(i=1, j=1, n=1$ ). During the calculation process, the plate's generatrix direction was slightly deformed, which can be ignored. Under the condition where only plate's normal deformation is caused, it can obtain:

$$
f_{1}\left(\pi^{4}+\pi^{2}+\mu T_{11}\right)+q_{1} \mu Q_{11}=-2 \mu+\frac{2 \mu}{l}\left(1+\frac{4 l^{2}}{\pi^{2}-4 l^{2}}\right) \sin (2 l) \sin \left(2 \theta_{0}\right)
$$


$f_{1} \mu K_{11}+q_{1}\left(\frac{41}{36} \pi^{4}-\frac{5}{8} \pi^{2}+\mu E_{11}\right)=-\frac{4 \mu}{l}\left[\frac{l^{2}}{\left(\frac{\pi}{2}\right)^{2}-4 l^{2}}-\frac{l^{2}}{\left(\frac{3 \pi}{2}\right)^{2}-4 l^{2}}\right] \times \cos (2 l) \sin \left(2 \theta_{0}\right)$

Normal displacement of a thin arched plate is expressed as:

$w=-\frac{L^{2}}{R}\left\{f_{1}[1+\cos (\pi \xi)]+q_{1} \sin (\pi \xi) \cos \left(\frac{\pi}{2} \xi\right)\right\}$

taking the formula above into (4), we have:

$$
M_{\theta \theta}=-\frac{D}{R^{2}} \frac{\partial^{2} w}{\partial \theta^{2}}=-\frac{D \pi^{2}}{R}\left[f_{1} \cos (\pi \xi)+\frac{5}{4} q_{1} \sin (\pi \xi)+q_{1} \cos (\pi \xi) \sin \left(\frac{\pi}{2} \xi\right)\right]
$$

The stress on upper surface of the plate is[9]:

$$
\sigma_{\theta \theta}=-\frac{6 D}{h^{2} R^{2}} \frac{\partial^{2} w}{\partial^{2} \theta}=-\frac{6 D}{h^{2} R^{2}}\left[f_{1} \cos (\pi \xi)+\frac{5}{4} q_{1} \sin (\pi \xi)+q_{1} \cos (\pi \xi) \sin \left(\frac{\pi}{2} \xi\right)\right]
$$

\subsection{The solution of thin arched plates in symmetrical transversal cross-flow}

When flowing direction is parallel to symmetric axis of plate's cross-section, the symmetric deformation occurs. Thus the equation (15) is changed into:

$$
\begin{aligned}
& \frac{\partial^{4} w}{\partial \theta^{4}}+\frac{\partial^{2} w}{\partial \theta^{2}}=\frac{R^{4}}{D}\left(p^{*}-p_{1}-p_{2}-w \frac{\partial p_{1}}{\partial r}-\frac{v}{r} \frac{\partial p_{1}}{\partial \theta}\right) \quad\left(r=R, \theta_{1}<\theta<\right. \\
& \left.\theta_{2}\right)
\end{aligned}
$$

Correspondingly, the formula (16) is simplified to:

$$
w=-\frac{L^{2}}{R} \sum_{i=1}^{I} f_{i}\left[1+(-1) i^{-1} \cos \left(i \pi \theta \frac{R}{L}\right)\right] \quad(i=1,2, \ldots, I)
$$

Fluid potential $\varphi_{1}$ and pressure $p_{1}$ remain the same. According to (17) and (18), however, $\varphi_{2}$ and $\mathrm{p}_{2}$ are expressed as:

$$
\begin{aligned}
& \varphi_{2}=\sum_{n=1}^{N}\left(\frac{R}{r}\right)^{n} A_{n} \cos (n \theta) \\
& p_{2}=\frac{2 \rho_{\infty} v_{\infty}^{2} l^{2}}{\pi} \sum_{n=1}^{N}\left\{\sum_{i=1}^{I} C_{n i} f_{i}[\cos ((n-1) \theta)-\cos ((n+1) \theta)]\right\} \quad(n=1,2, \ldots, N ; i=
\end{aligned}
$$

$1,2, \ldots, I)$

where

$$
\begin{aligned}
& A_{n}=\frac{2 v_{\infty} L^{2}}{n \pi R} \sum_{i=1}^{I} C_{n i} f_{i} \\
& C_{n i}=(i \pi)^{2} n\left[\frac{\sin \beta_{n+1}}{(n+1)\left(\beta_{n+1}^{2}-i^{2} \pi^{2}\right)}-\frac{\sin \beta_{n-1}}{(n-1)\left(\beta_{n-1}^{2}-i^{2} \pi^{2}\right)}\right]
\end{aligned}
$$

Assuming $\mathrm{i}=1, \mathrm{n}=1$ and taking the above formulas into (19), we obtain:

$$
\begin{aligned}
& f_{i}\left\{\pi^{4}+\pi^{2}+C_{11} \frac{4 \pi}{\mu}\left[-2 l^{2}+\left(1+\frac{4 l^{2}}{\pi^{2}-4 l^{2}}\right) \sin (2 l)\right]-12 \mu+\frac{4 \mu}{l R}\left(\frac{3}{2}+\frac{8 l^{2}}{\pi^{2}-4 l^{2}}\right) \sin (2 l)\right\} \\
& = \\
& \mu\left[-2+\frac{2}{l}\left(1+\frac{4 l^{2}}{\pi^{2}-4 l^{2}}\right) \sin (2 l)\right]
\end{aligned}
$$

\section{Verification with an example}

Assuming that the thickness of a thin arched plate is $\mathrm{h}=2 \times 10^{-3} \mathrm{~m}$, the radius is $\mathrm{R}=0.85 \mathrm{~m}$, the attack angle $\theta_{0}=13^{\circ}$, the arc length is $2 \mathrm{~L}=0.84 \mathrm{~m}$, elastic modulus $\mathrm{E}=210 \mathrm{GPa}$, the Poisson's ratio $v=0.23$ and the pressure and the intensity of incompressible ideal fluid at infinite distance are $\mathrm{p}_{\infty}=1000 \mathrm{~Pa}, \rho_{\infty}=1000 \mathrm{~kg} / \mathrm{m}^{3}$, then the following curves can be obtained by Matlab.

If copper, aluminum and low carbon steel are respectively chosen as materials for a thin arched plate and submerge them into an ideal fluid, the normal displacement occurred is shown in Fig. 3. The results show that the maximum displacement appears at $\theta=9.6^{\circ}$ rather than $\theta=13^{\circ}$.

With other conditions unchanged, normal displacement of plates which are made up of different materials varies with thickness, arc length and fluid speed, shown in Fig.4, Fig.5 and Fig.6. The results reveal that less thickness, longer arc-length and higher fluid speed cause larger displacement in plates.

Fig. 7 reflects the relationship between normal displacement and attack angles. The results show that the smaller attack angle is, the larger normal displacement is. However, attack angles should be 
less than $30^{\circ}$ on account of geometric dimension. When flow direction is parallel to normal line of mid-surface (that is, $\theta_{0}=0$ ), the maximum displacement is $1.64 \times 10^{-3} \mathrm{~m}$; when $\theta_{0}=13^{\circ}$, the maximum normal displacement is $1.332 \times 10^{-3} \mathrm{~m}$, which occurs at $\theta=9.6^{\circ}$.

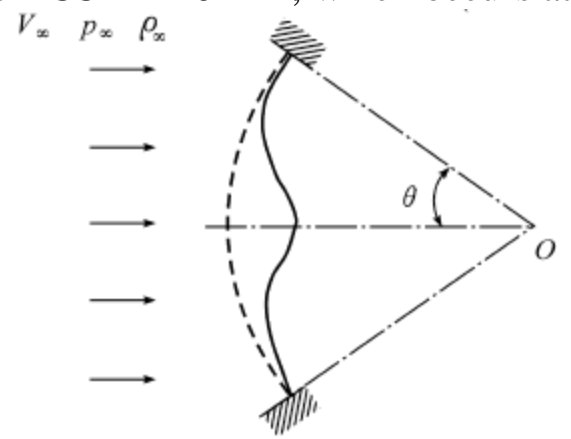

Fig. 2 Symmetrical transversal cross-flow on thin arched shell

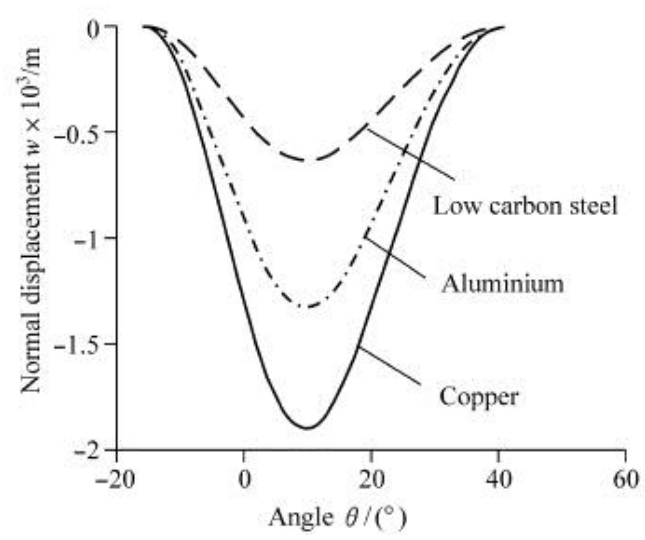

Fig. 3 Normal displacement curve of different materials

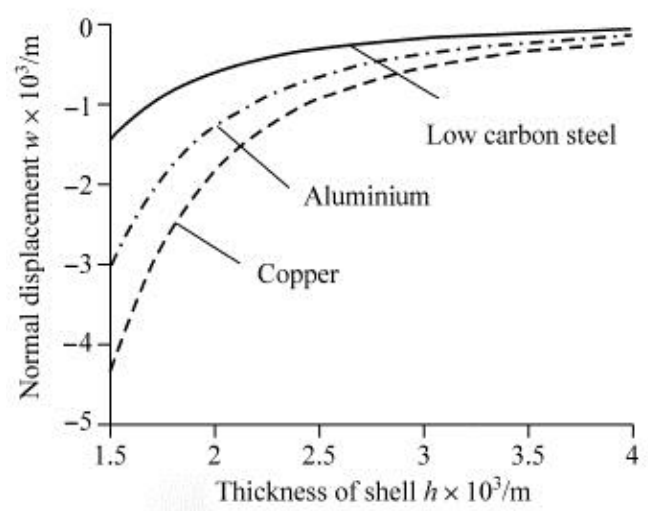

Fig. 4 Curve of normal displacement and thickness

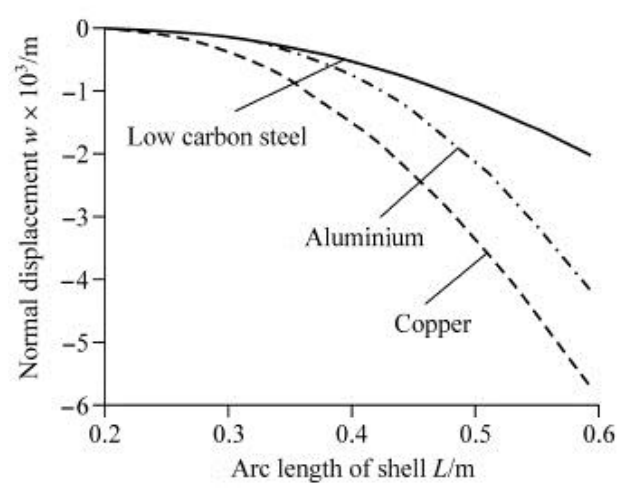

Fig. 5 Curve of normal displacement and arc length 


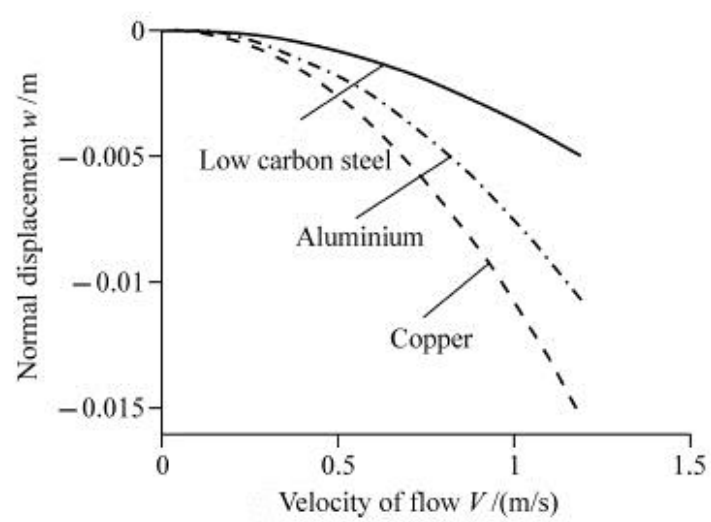

Fig. 6 Curve of normal displacement and velocity of flow

With other conditions unchanged, normal displacement of plates which are made up of different materials varies with thickness, arc length and fluid speed, shown in Fig.4, Fig.5 and Fig.6. The results reveal that less thickness, longer arc-length and higher fluid speed cause larger displacement in plates.

\section{Conclusion}

The displacement, bending moment and stress of a thin arched plate vary, though in the same flow regime, with plate's positions in fluids, whose values and positions can be obtained by the analysis above. The impact of materials, thickness, arc-length and velocity of fluid on deformation and stress were discussed. The more attack angle increases, the less normal displacement is. The maximum displacement happens at $\theta_{0}=0$, that is, flow direction is along symmetric axis of a plate.

\section{References}

[1] BAI XiangZhong, HAO YaJuan. Advances in nonlinear hydroelasticity [J]. Advances in Mechanics, 2008, 38(5) : 545-560( In Chinese) .

[2] HAO YaJuan, YANG Yang, BAI XiangZhong. Analysis of deformation and stress of elastic plate in a tank under the influence of fluid [J]. Journal of Mechanical Strength, 2009, 31( 2) : 250255( In Chinese) .

[3] ZHANG SuXia, LIU XiJun, JIA QiFen, et al. Experiment modal analysis on rectangular elastic fluid-filled shell [J] . Journal of Mechanical Strength, 2004, 26( 6) : 615-619(In Chinese) .

[4] Trjsseling A S. Exact solution of linear hyperbolic four-equation system in axial liquid-pipe vibration [J] . Journal of Fluid and Structures, 2003, 18: 179-196.

[5] LI YaZhi, CHEN Gang. Numerical simulation of liquid-filled tank response to projectile impact [J] . Journal of Mechanical Strength, 2007, 29(1) : 143-147( In Chinese) .

[6] QIN TaiYan, LI Jie, CHANG TianChun, DUAN MengLan. Study of fracture and reliability assessment of deepwater risere [ J] . Journal of Mechanical Strength, 2004, 26(Suppl. 1) : 063 066( In Chinese) .

[7] Xia L J, Wu W G, Weng C J, Jin X D. Analysis of fluid-structure-coupled vertical vibration for high-speed ships[ J] . Journal of Ship Mechanics, 2000, 4(3): 43-48.

[8] [8]CHENG GengDong, LIHaiTao, RUAN ShiLun. Free vibration characteristics and stability analysis of shiplift system [J]. Journal of Mechanical Strength, 2005, 27(3): 276-281(In Chinese) .

[9] Alberto Figueroa C, Irene E, Vignon-Clementel, Kenneth E Jansen, Thomas J R Hughes, Charles A Taylor. A coupled momentum method for modeling blood flow in three-dimensional deformable arteries[J] . Computer Methods in Applied Mechanics and Engineering, 2006, 195 41-43) : 5685-5706. 